\title{
Off-Resonance SERS Nanoprobe-Targeted Screen of Biomarkers for Antigens Recognition of Bladder Normal and Aggressive Cancer Cells
}

Yao-Tzu Yang, ${ }^{\dagger a, b}$ I-Ling Hsu, ${ }^{\dagger b}$ Ting-Yu Cheng, ${ }^{\dagger a}$ Wen-Jeng Wu, ${ }^{c}$ Chien-Wei Lee, ${ }^{b}$ Tsung-Ju Li, ${ }^{b}$ Chun In Cheung, ${ }^{\mathrm{b}}$ Yu-Cheng Chin, ${ }^{\mathrm{b}}$ Hsiao-Chien Chen, ${ }^{\mathrm{d}}$ Yi-Chun Chiu, ${ }^{\mathrm{e}, \mathrm{f} *}$ Chih-Chia Huang,,${ }^{\mathrm{b}}{ }^{*}$ and Mei-Yi Liao ${ }^{a^{*}}$

${ }^{a}$ Department of Applied Chemistry, National Pingtung University, Pingtung 90003, Taiwan

${ }^{b}$ Department of Photonics, National Cheng Kung University, Tainan 70101, Taiwan

${ }^{\mathrm{c}}$ Graduate Institute of Medicine, College of Medicine, Kaohsiung Medical University, Kaohsiung 80708, Taiwan

${ }^{\mathrm{d}}$ Center of Applied Nanomedicine, National Cheng Kung University, Tainan 70101, Taiwan

e Division of Urology, Department of Surgery, Zhong Xiao Branch, Taipei City Hospital, Taipei 11556, Taiwan

${ }_{\mathrm{f}}^{\mathrm{f}}$ Department of Urology, School of Medicine, National Yang-Ming University, Taipei City 11221, Taiwan

$\dagger$ These authors contribute equally to this work.

* Corresponding authors:

Dr. Yi-Chun Chiu, Email: DAM15@tpech.gov.tw; c333732@gmail.com

Prof. Chih-Chia Huang, E-mail: c2huang@mail.ncku.edu.tw; huang.chihchia@gmail.com Prof. Mei-Yi Liao, E-mail: myliao@mail.nptu.edu.tw 


\section{MATERIALS and METHODS}

Materials. Hydrogen tetrachloroaurate(III) trihydrate $\left(\mathrm{HAuCl}_{4} \cdot 3 \mathrm{H}_{2} \mathrm{O}\right), 99.9 \%$ (Alfa Aesar), poly(styrene-alt-maleic acid) sodium salt solution (PSMA) (Aldrich), sodium chloride ( $\mathrm{NaCl})$ (Fisher chemical), silver nitrate $\left(\mathrm{AgNO}_{3}\right)$ (Fisher chemical), nitric acid $\left(\mathrm{HNO}_{3}\right)$ (Fisher chemical), HS-PEG3500-NH2 (JenKem Technology), sodium hyaluronate (Flamegold Material Corporation), human transferrin (Sigma), anti-EGF receptor clone LA22 antibody (EMD Millipore), 4carboxyphenylboronic acid (Aldrich), and folic acid (Sigma) were purchased for use without further purification.

Synthesis of Ag Nanoparticles. To prepare Ag nanoparticles, polyvinylpyrrolidone (PVP) $(0.10 \mathrm{~g})$ and $\mathrm{AgNO}_{3}(0.025 \mathrm{~g})$ were dissolved in $10 \mathrm{~mL}$ of ethylene glycol. The solution was heated at $160{ }^{\circ} \mathrm{C}$ for 1.5 hours (hrs). Afterward, the green-yellow colloidal solution was cooled to room temperature. The resulting solution was reserved for the following reactions in the preparation of 3D AuAg nanohollows.

To fabricate the 3D AuAg nanohollows developed by our group, ${ }^{1} \mathrm{HAuCl}_{4}$ solution $(4 \mathrm{~mL}, 0.08$ $\mathrm{mM}$ ) was dropwise added into a mixture of $250 \mu \mathrm{L}$ of PVP-coated Ag colloidal solution (1.35 mM) and PSMA solution $(1.2 \mathrm{~g} / \mathrm{mL})$. The resulting solution presented a yellow color, suggesting formation of solid-core nanoparticles. After the injection of $\mathrm{HNO}_{3}(0.22 \mathrm{M})$, the color of the solution began to change from yellow to blue. To prepare the Raman reporter, $4 \mathrm{~mL}$ of 3D AuAg nanohollows was reacted with $0.1 \mathrm{~mL}$ of 4 -ATP. After $12 \mathrm{~h}$ of reaction time, a repeated process of 
centrifugation and washing with deionized water was employed to purify the 4-ATP-immobilized AuAg nanohollows, and then, the nanohollows were transferred for the subsequent surface modification.

Synthesis of Ligands/4-ATP-Modified AuAg Nanohollows. To prepare the ligand/4-ATPmodified 3D AuAg nanohollows, $200 \mu \mathrm{L}$ of 4-ATP-immobilized AuAg nanohollows (200 ppm[Ag]) was reacted with HS-PEG-NH 2 polymer solution $(200 \mu \mathrm{L}$ at $2 \mathrm{mg} / \mathrm{mL})$ at $4{ }^{\circ} \mathrm{C}$ for $120 \mathrm{mins}$, allowing the SH groups of the PEG polymer to chemically bind to the surface of the AuAg nanohollows, exposing their primary amine groups at the surface. After purification with a centrifugation and re-dispersion process, the PEG/ATP-modified AuAg nanohollows were subsequently re-suspended in the pre-mixed ligand solution $(0.85 \mathrm{~mL})$, including $0.05 \mathrm{~mL}$ of 0.025 $\mathrm{mM}$ small molecules (i.e., FA, HA, and CPBA), $7 \mathrm{mg} / \mathrm{mL} \mathrm{N}$-(3-dimethylaminopropyl)-N'ethylcarbodiimide hydrochloride (EDC), and $7 \mathrm{mg} / \mathrm{mL}$ N-hydroxysuccinimide (NHS). After a 40 min bath with sonication at $4{ }^{\circ} \mathrm{C}$, the sample solution was centrifuged at $10,000 \mathrm{rpm}$ for 20 min to remove the excess molecules, and a repeated centrifugation/washing process with PBS solution was performed to purify the ligand/4-ATP-modified 3D AuAg nanohollows. A similar synthesis method was performed to prepare TF/ATP-modified AuAg nanohollows and anti-EGFR/ATP-modified AuAg nanohollows using $1 \mathrm{mg} / \mathrm{mL}$ TF and $20 \mu \mathrm{g} / \mathrm{mL}$ anti-EGFR, respectively.

SERS Measurement of AuAg Nanohollows. For SERS detection, a $10 \mu \mathrm{L}$ sample of the CPBA/4-ATP-modified AuAg nanohollows ( 25 ppm $[\mathrm{Ag}])$ was carefully transferred onto silicon 
substrates and then subjected to micro-Raman spectroscopy using a $785 \mathrm{~nm}$ laser (DPSSL Driver II, $3.4 \mathrm{~mW}$ ) and an MRS-iHR320 modular Raman system equipped with an Olympus BX53 microscope and a 40X objective lens. An acquisition time of $5 \mathrm{~s}$ was used for Raman measurements at each point. The smoothing method of Savitzky-Golay filter in Origin 8.0 software was applied to the data for improving the signal-to-noise ratio by fitting adjacent data points with a polynomial of degree 2 .

Cell Culture. Human urinary bladder transitional cell carcinoma (T24 and RT4) cells, a human immortalized uroepithelial cell line (SV-HUC-1), and a mouse embryo fibroblast cell line (NIH/3T3) were obtained from the Bioresource Collection and Research Center (BCRC), Hsinchu, Taiwan. The MB49 cancer cell line (murine transitional cell carcinoma) was kindly provided by Dr. WenJeng Wu (Department of Urology, Kaohsiung Medical University Hospital, Kaohsiung Medical University, Kaohsiung, Taiwan). T24 (HTB-4, grade III) and RT4 (HTB-2, grade I) cells were cultured in McCoy's 5A medium. SV-HUC-1 cells were cultured in Ham's F- 12K (Kaighn's) medium. NIH/3T3 cells were cultured in Dulbecco's Modified Eagle Medium (DMEM). MB49 cells were cultured in RPMI-1640 medium. All media were supplemented with $10 \%$ fetal bovine serum (FBS), 100 units $/ \mathrm{mL}$ penicillin, and $100 \mu \mathrm{g} / \mathrm{mL}$ streptomycin. All culture media, FBS, and supplements were obtained from Gibco (Thermo Fisher Scientific, Waltham, MA, USA).

Detection of Suspended T24 Cells with CPBA/4-ATP-Modified AuAg Nanohollows. To prevent non-specific interactions, $1 \times 10^{4} \mathrm{~T} 24$ cells were suspended in $1 \mathrm{~mL}$ of $1 \%$ bovine serum 
albumin (BSA) on ice for 30 mins. After centrifugation at $1200 \mathrm{rpm}$, the supernatant was discarded, and the cell pellet was resuspended in urine solution including CPBA/4-ATP-modificed AuAg nanohollows $(20 \mathrm{ppm}[\mathrm{Ag}])$. The mixture was incubated for $15 \mathrm{mins}$ at $4{ }^{\circ} \mathrm{C}$. Afterward, we employed a repeated centrifugation and washing process with PBS to remove the non-adhered CPBA/4-ATPmodificed AuAg nanohollows. Subsequently, the cell pellet was resuspended in $10 \mu \mathrm{L}$ of urine solution, transferred onto $\mathrm{Si}$ substrates, and subjected to micro-Raman spectroscopy. The customized micro-Raman spectroscopy instrument was built by integrating a Jobin-Yvon LabRAM high-resolution Raman spectrometer (Horiba iHR 320) with Olympus BX3 microscopes to obtain individual Raman signals at different positions in the cellular images. A 20X objective lens was used to collect point-to-point signals with a computer-controlled $\mathrm{x}, \mathrm{y}$-stage in $4.0 \mu \mathrm{m}$ increments using a Raman microscope. Each data point was measured with a $785 \mathrm{~nm}$ laser $(3.4 \mathrm{~mW})$ and a 1$\mathrm{s}$ acquisition time. Maps were generated by taking 2 spectra per $\mu \mathrm{m}$ in both the $\mathrm{x}$ and $\mathrm{y}$ directions, with specific Raman signals of 4-ATP forming a 2-dimensional (2-D) map. All spectral controls, manipulations, and data analyses were performed using LabRAM systems.

Raman Mapping Microscopy of Live Cells. T24 cells $\left(1 \times 10^{4}\right)$ were seeded into the chamber slide. After incubation at $37{ }^{\circ} \mathrm{C}$ for $24 \mathrm{hrs}$, the culture medium was removed. Then, $200 \mu \mathrm{L}$ of medium including CPBA/4-ATP-modified 3D AuAg nanohollows (20 $\left.\mathrm{ppm}_{[\mathrm{Ag}]}\right)$ was added into the seeded cells and co-cultured at $37^{\circ} \mathrm{C}$ for $1 \mathrm{hr}$. The excess AuAg nanohollows were washed away with PBS buffer solution. The particle-treated cells were then subjected to a micro-Raman system. 
The laser power was $3.4 \mathrm{~mW}$, the integration time was $1 \mathrm{~s}$, and a $60 \mathrm{X}$ objective lens was used for SERS mapping.

Photothermal Effect on Cell Morphology. T24 cells were pre-cultured in a $35 \mathrm{~mm}$ dish (1.5 x $10^{5}$ cells per well) for 1 day. After the old medium was replaced with $0.2 \mathrm{~mL}$ of PBS, T2 4 cells were then subjected to micro-Raman spectroscopy and irradiated with a $785 \mathrm{~nm}$ laser $(1,3.4,4,16$, $30 \mathrm{~mW}$ ) through a $40 \mathrm{X}$ objective during a recording period of 60 mins. The morphological changes of the T24 cells were observed under a microscope.

Photothermal Effect on Cell Viability. The CPBA/4-ATP-modified AuAg nanohollowmediated photothermal effect on T24 cell viability was evaluated by MTT assay. In brief, T24 cells were seeded at a density of 8000 cells/well in 96-well plates and incubated for $24 \mathrm{hrs}$. Then, 100 $\mu \mathrm{L}$ of $35 \mathrm{ppm}_{[\mathrm{Ag}]} \mathrm{CPBA} / 4-\mathrm{ATP}-$ modificed 3D AuAg nanohollows dispersed in the culture media was added to each well and incubated for $1 \mathrm{hr}$. After incubation, cells were irradiated with a 785$\mathrm{nm}$ wavelength laser $(100 \mathrm{~mW})$ to cover the whole area of each well in the 96 -well plates for 5-10 mins. Then, the nanohollow-containing medium was replaced with fresh medium, and the cells were incubated for another $24 \mathrm{hrs}$. After the 24-hr incubation, MTT reagent was added into each well, and cells were further incubated for $1 \mathrm{hr}$. The medium was removed, and DMSO was added into each well to dissolve formazan crystals. Finally, the absorbance was measured at $565 \mathrm{~nm}$ with a microplate reader (Synergy H1, BioTek). Cell viability was reported as a percentage calculated as the ratio between the treated groups and the control group (without nanohollow and light 
treatments).

Mice. C57BL/6 mice were purchased from the National Laboratory Animal Center (Taipei, Taiwan). All animal studies were performed according to protocols approved by the Institutional Animal Care and Use Committee of Kaohsiung Medical University.

Orthotopic Murine Bladder Cancer Model. 12-week-old female C57BL/6 mice were used in orthotopic murine bladder cancer experiments. Mice were anesthetized with $2 \%(\mathrm{v} / \mathrm{v})$ isoflurane and maintained with inhalation of oxygen. Mouse bladders were catheterized using $24 \mathrm{G}$ permanent venous catheters (Terumo Corporation, Tokyo, Japan) and the bladder residual urine was removed by massaging the bladder with finger. Then mouse bladders were sequentially rinsed with $100 \mathrm{uL}$ of $0.1 \mathrm{~N} \mathrm{HCl}$ and $100 \mathrm{uL}$ of $0.1 \mathrm{~N} \mathrm{KOH}$, and installed with $80 \mathrm{uL}$ of $0.125 \%$ trypsin for a 15 -minute incubation. After sterile saline washing, $100 \mathrm{uL}$ of $5 \times 10^{5} \mathrm{MB} 49$ cells was installed into mouse bladder cavities of mice and retained for 2 hours. One week after implantation, the ultrasound imaging was performed using a VisualSonics Vevo 770 High-Resolution Imaging System (Visual Sonics Inc, Toronto, Ontario, Canada) with a $25 \mathrm{MHz}$ probe in B mode to identify the bladder tumor region before subsequent $\mathrm{AuAg}$ nanohollow and NIR light treatments.

In Vivo Treatment with AuAg Nanohollows and NIR Light. $100 \mathrm{uL}$ of $100 \mathrm{ppm}[\mathrm{Ag}]$ 4ATP/PEG-modified AuAg nanohollows and 4-ATP/CPBA-modified AuAg nanohollows were instilled into the bladder cavity of mice in orthotopic murine bladder cancer model and retained for $1 \mathrm{hr}$. Next, the bladder was gently washed twice with sterile PBS to remove non-specific binding 
of nanoparticles to the bladder. Mice were sacrificed immediately and the bladder was resected for further SERS detection. The 785nm laser was carefully directed towards the organ surface just above the murine bladders with the guidance of visible light. To determine the present of MB49 cells in the murine bladder cavity, Raman signals of the AuAg nanohollows bound to murine bladders were detected by employing the micro-Raman spectroscopy equipped with a 785-nm laser $(3.4 \mathrm{mV})$ and a $30 \mathrm{~s}$ acquisition time.

In in vivo photothermal experiment, mice bladder tumors bound by CPBA/4-ATP-modified 3D AuAg nanohollow were irradiated with a NIR laser at a wavelength of $808 \mathrm{~nm}$ at a power density of $1.75 \mathrm{~W} / \mathrm{cm}^{2}(0.365 \mathrm{~mW})$ for 10 minutes at a fixed distance from the tip of laser fiber to the skin surface $(1 \mathrm{~cm})$. The changes in mouse body temperature were measured with thermometer (Fluke Corporation, WA, USA).

Characterizations. Transmission electron microscopy (TEM, Hitachi H7500 TEM instrument at $80 \mathrm{kV}$ ) was used to determine the structures of the nanomaterials. The absorption spectra of the AuAg nanohollows were measured using a V-730 UV-Vis spectrophotometer from Jasco (USA). To prepare same particle concentration for the UV-visible measurement, purified samples were collected from the same volume in a single vial solution of AuAg nanohollows and 3D AuAg nanohollows with the same Ag NPs template as a starting material. We assumed no loss of particle number after a repeated centrifugation and re-dispersion process. The $\mathrm{Au}$ and $\mathrm{Ag}$ concentrations of the Au/Ag-based materials were quantified by AAS (SensAA GBC, Australia). 
The particle sizes and zeta potentials (HORIBA, Ltd., Japan) of the Au/Ag-based samples dispersed in aqueous solution were measured. In the Raman analysis, the samples were placed onto silicon substrates and were subjected to micro-Raman spectroscopy equipped with a 785-nm laser (DPSSL Driver II, $10 \mathrm{~mW}$ ). 20X, 40X, and $60 \mathrm{X}$ objective lens were applied for the experiments of suspended T24 cell detection, photothermal effect, and Raman mapping of live cells, respectively. All spectral controls, manipulations, and data analyses were performed using LabRAM systems. 


\section{SCHEMES}

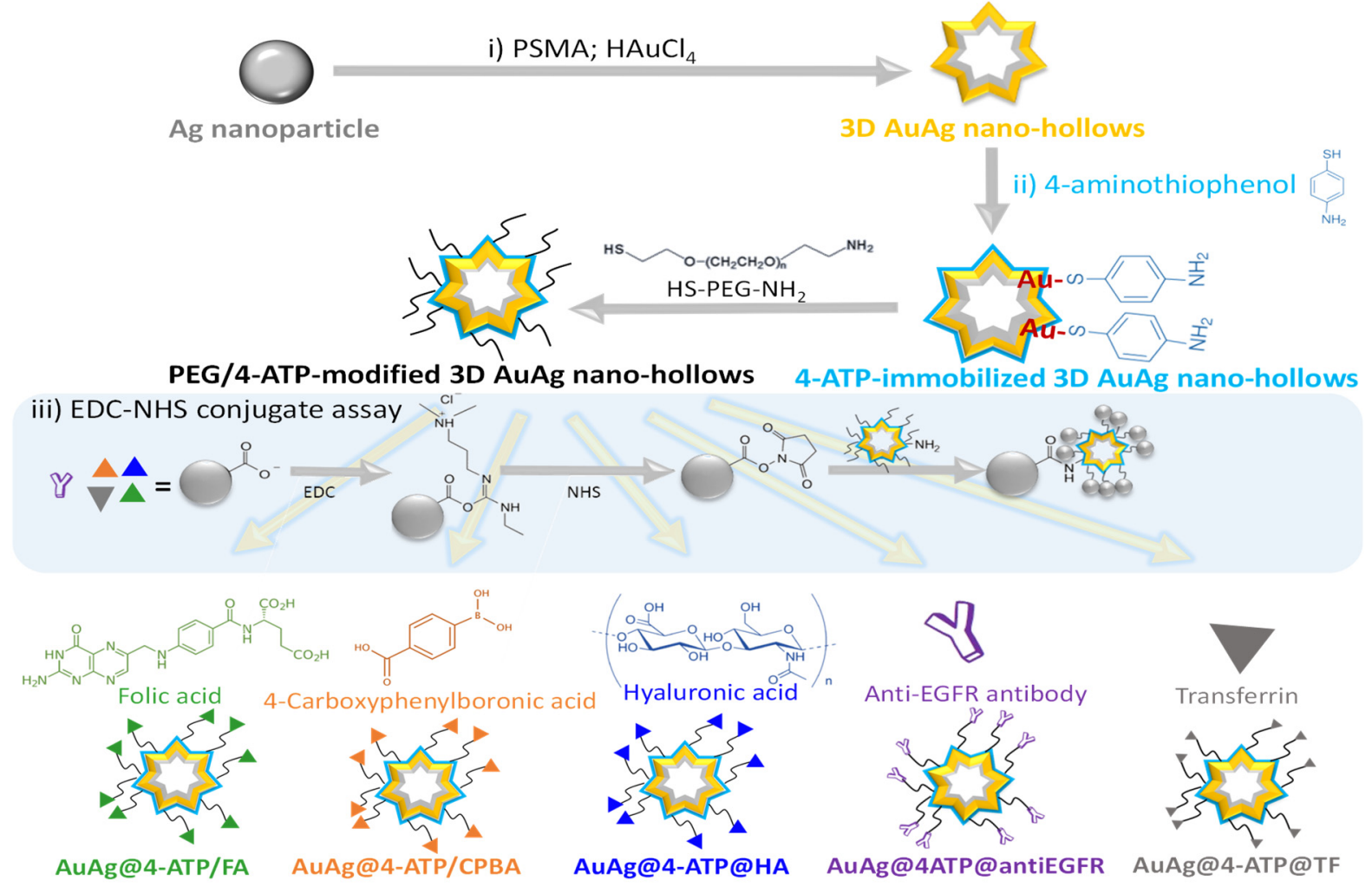

Scheme S1. Schematic diagram for i) the synthesis of 3D AuAg nanohollows and ii) 4-ATP immobilization, followed by iii) functionalization with biomolecules via EDC/sulfo-NHS ester chemistry. 
a)

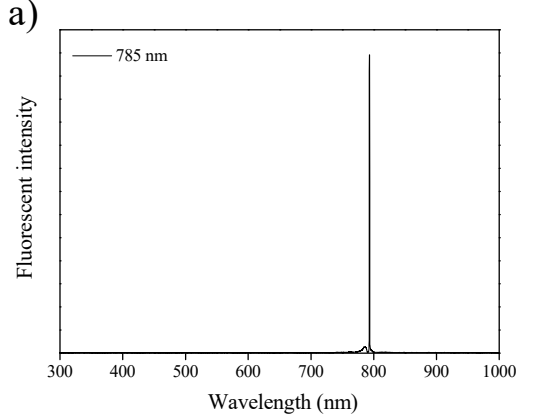

d)

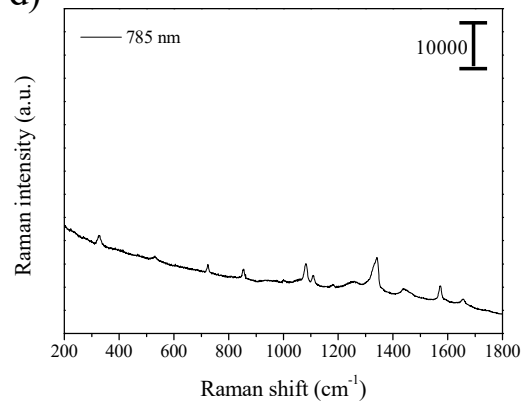

b)

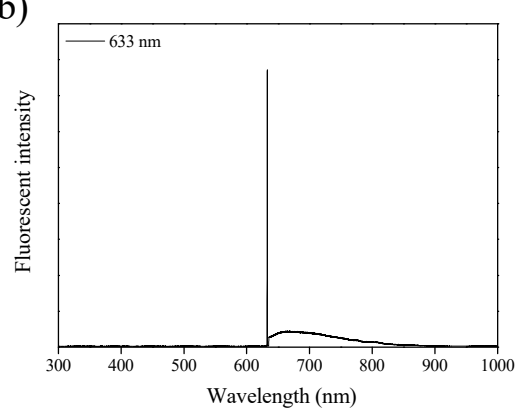

e)

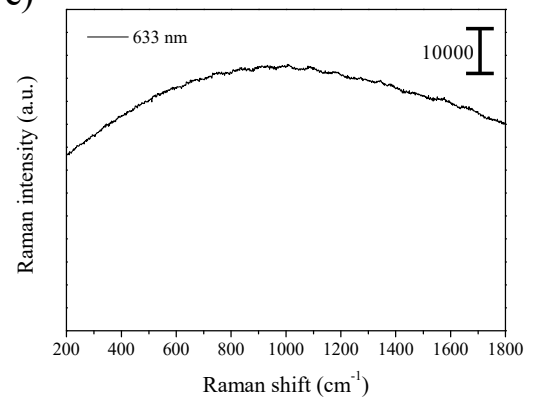

c)

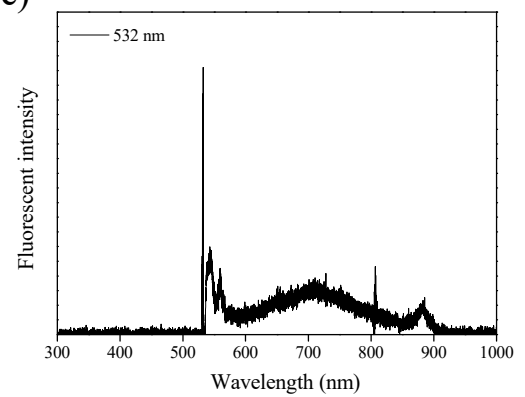

f)

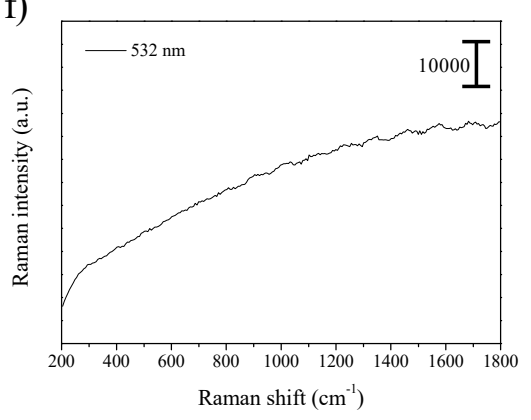

Figure S1. The fluorescent spectra of normal bladder tissues excited by using a) $785 \mathrm{~nm}-$, b) 632.8 nm-, and c) $532 \mathrm{~nm}$-laser light wavelengths. The SERS spectra of bladder cancer tissues with 100 ppm $_{[\mathrm{Ag}]}$ CPBA/4-ATP-modified AuAg nanohollows excited by using d) $785 \mathrm{~nm}-$, e) $632.8 \mathrm{~nm}$-, and f) $532 \mathrm{~nm}$-laser light wavelengths. 
It can be seen that very little change was observed in the SPR band position of the 3D AuAg nanohollows in the different solution systems with aging time on the basis of the UV/visible spectra (Figure S2a). The two high absorption peaks at $276 \mathrm{~nm}$ and $559 \mathrm{~nm}$ were contributed from DMEM medium (Figure S3). In addition, the absorption intensity of the as-synthesized 3D AuAg nanohollows SPR band slightly decreased by $\sim 2 \%$ after 14 days of storage time, even for colloids exposed to a high-salt buffer solution. This result indicated that the 3D AuAg nanohollow was a robust carrier for SERS molecules or biomarkers to protect against salt-induced aggregation. Moreover, the SERS spectra of 4-ATP-immobilized 3D AuAg nanohollows (without PEG coating) in different solutions were nearly consistent in their Raman peak intensity at $1079 \mathrm{~cm}^{-1}$ during 14 days of aging time (Figure S2c). The temperatures of these 3D AuAg nanohollow solutions (35 $\operatorname{ppm}_{[\mathrm{Ag}]}$ ) were increased to $\sim 43.9$ and $\sim 41.8{ }^{\circ} \mathrm{C}$ after 10 minute (min) under $100 \mathrm{~mW}$ NIR laser irradiation at $785 \mathrm{~nm}$ and $808 \mathrm{~nm}$, respectively (Figure S2d). The photothermal conversion effect of the 3D AuAg nanohollow solution was very stable in the different dispersed solution phases with respect to the steric protection by PSMA polymer around the nanoparticle. Similar temperature rising values were determined for the samples in PBS and medium solution after 14 days of aging time.

a)
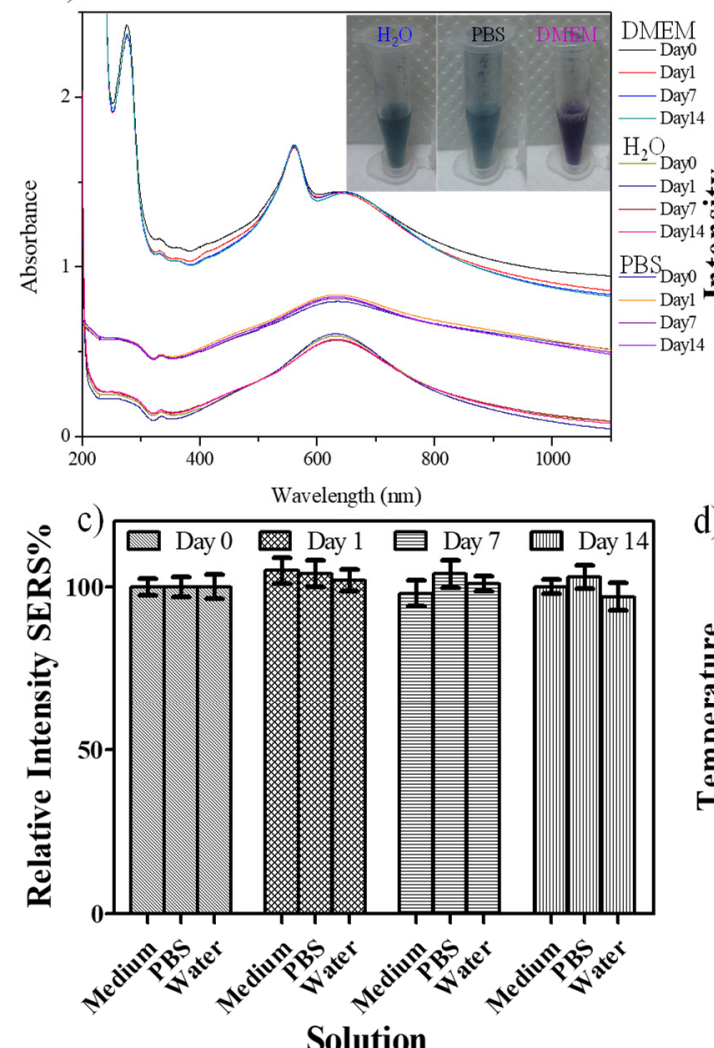

b)
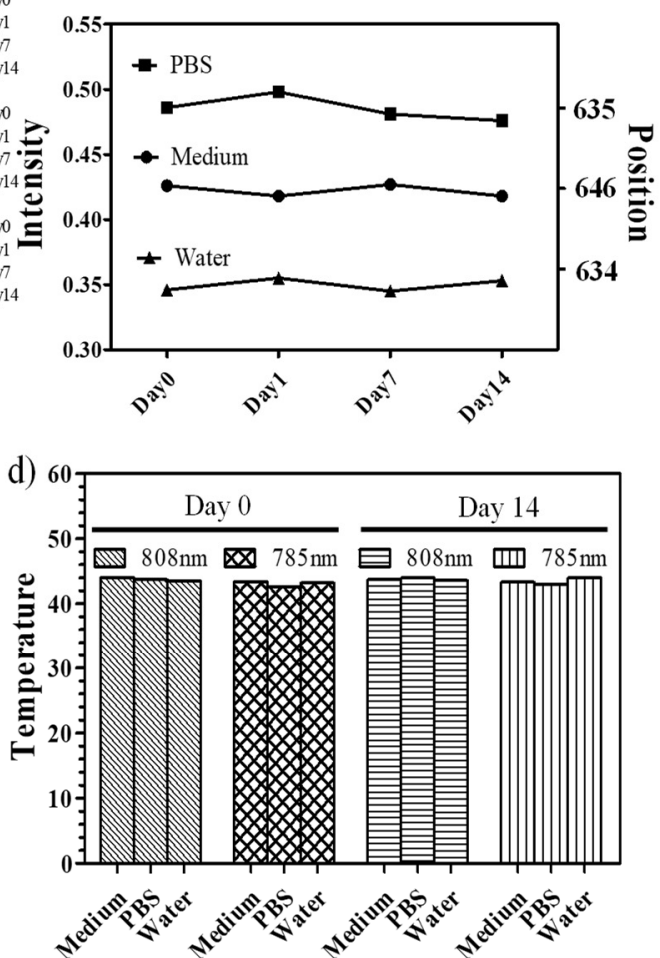

Solution

Figure S2. a) UV-visible spectra and b) the corresponding absorption intensity and band position plot versus the aging time of as-synthesized AuAg nanohollows in water, PBS, and DMEM solutions. c) SERS peak at $1079 \mathrm{~cm}^{-1}$ recorded with aging time and d) photothermal converted temperature as a function of the irradiation time of the 4-ATP-immobilized AuAg nanohollows (without PEG coating) stored in water, PBS, and DMEM solutions. 


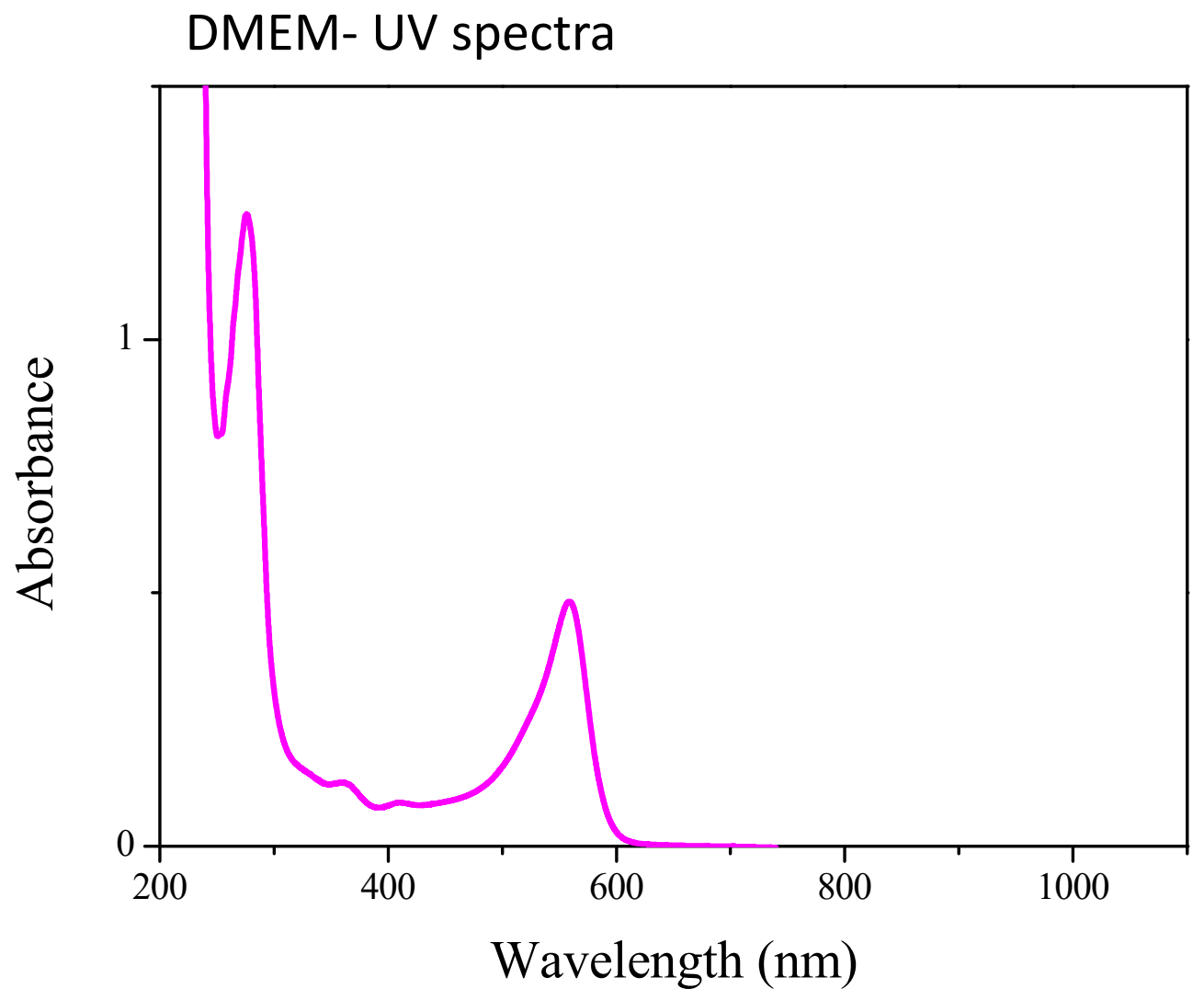

Figure S3. UV-visible spectra of DMEM medium. 
a) 0 min

$1 \min$

$3 \mathrm{~min}$

$5 \mathrm{~min}$

$10 \mathrm{~min}$
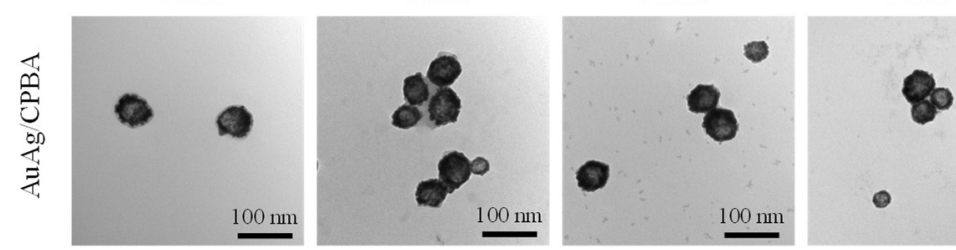

b)

c)
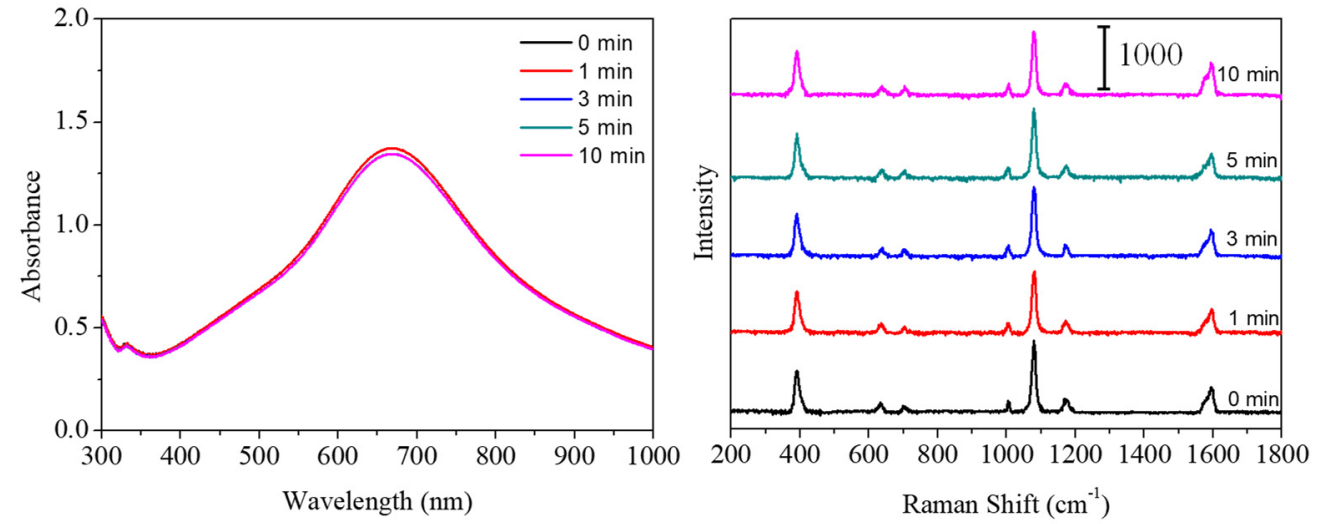

Figure S4. a) TEM images, b) UV-visible spectra, and c) SERS spectra of CPBA/4-ATP-modified $\mathrm{AuAg}$ nanohollows which received $16 \mathrm{~mW}$ of $785-\mathrm{nm}$ laser power from 0-10 min. The sample concentration was $25 \mathrm{ppm}_{[\mathrm{Ag}]}$ for all analysis. 


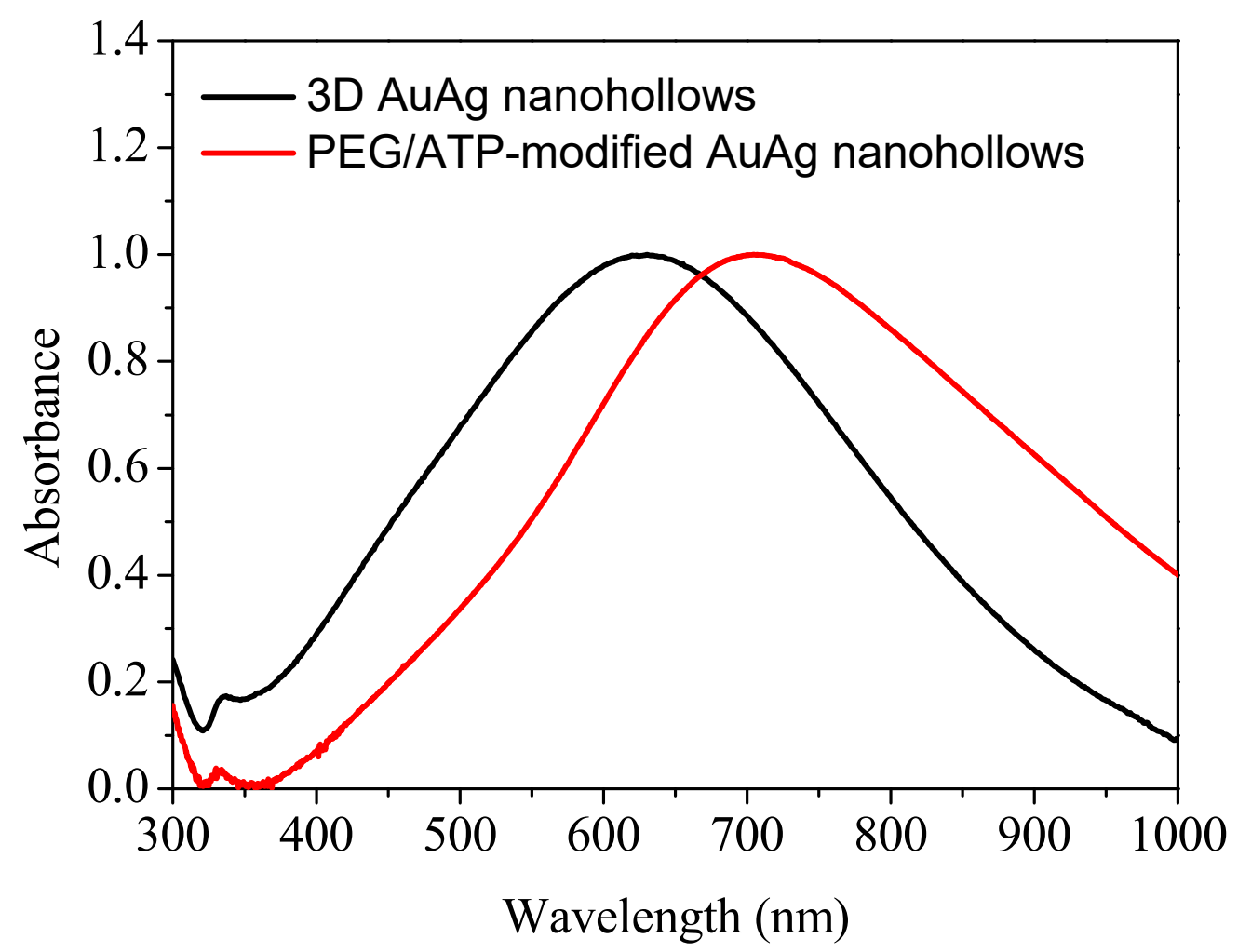

Figure S5. UV-visible spectra of 3-D AuAg nanohollows and PEG/ATP-modified AuAg nanohollows. 


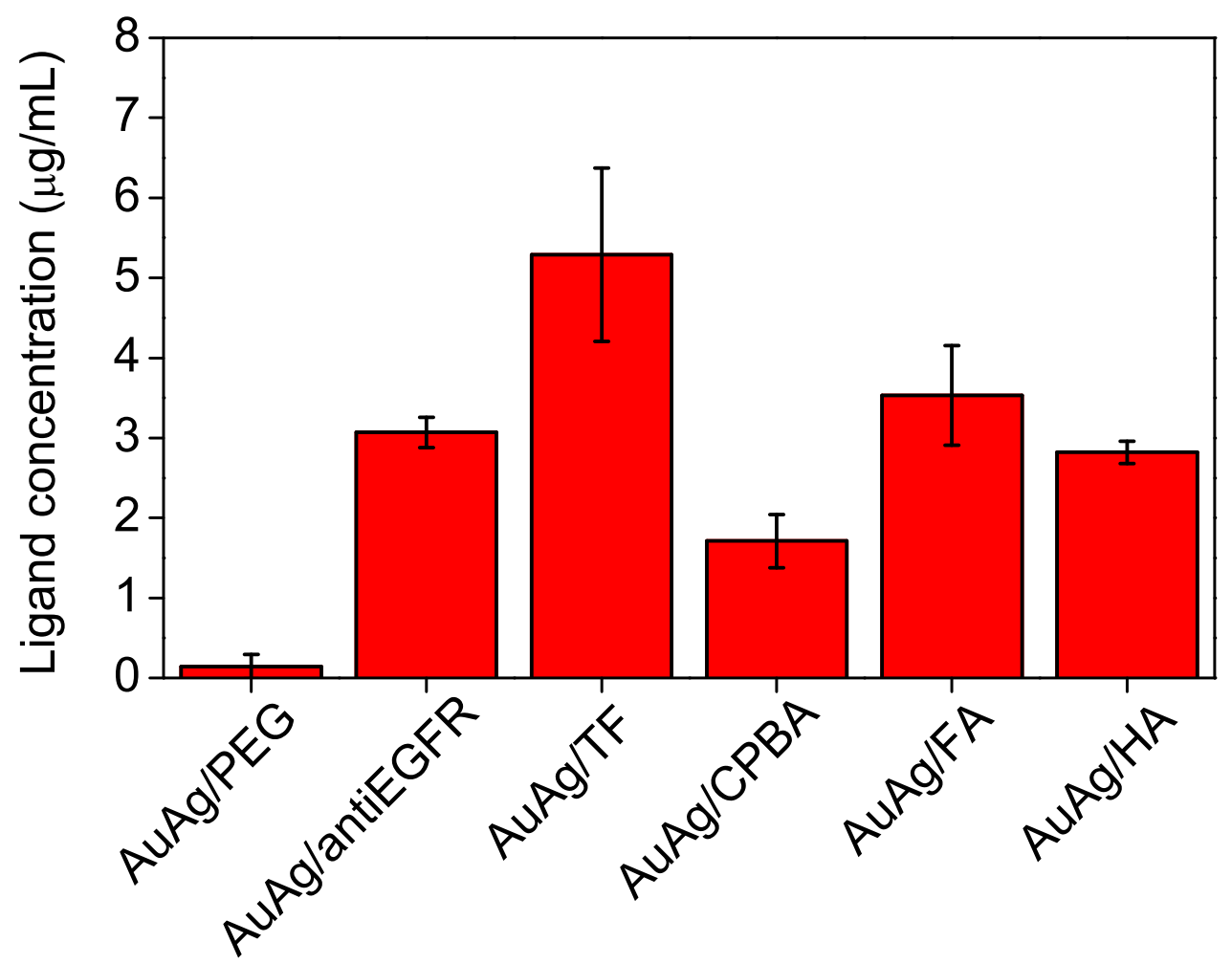

Figure S6. Quantitation of different ligands-conjugated to 4-ATP-immobilized AuAg nanohollows. BCA method was employed to measure the protein amount on the 4-ATP/PEG-immobilized AuAg nanohollows, anti-EGFR/4-ATP-immobilized AuAg nanohollows, and TF/4-ATP-immobilized AuAg nanohollows. The conjugation amount of CPBA, FA, and HA on the 4-ATP-immobilized AuAg nanohollows was determined by using Lambert-Beer's Law to compare the absorbance at $\lambda_{\max }$ for each collected supernatant and corresponding control group. The sample concentration was $1 \mathrm{ppm}_{[\mathrm{Ag}]} 4$-ATP-immobilized AuAg nanohollow for all analysis. 
High-resolution X-ray photoelectron spectroscopy (XPS) spectra was applied to analyze the coating of the 4-ATP and CPBA molecules in the formation of the CPBA/4-ATP modified AuAg nanohollows. Figure S7a and S7b show the spectra S 2P and B 1S regions, respectively. ${ }^{2,3}$ XPS measurement determined at the surface of the 3D AuAg nanohollows has $\sim 4.2 \% \mathrm{~S}$ from 4-ATP and $\sim 1.8 \%$ B from CPBA. Noted that doublet peak at $\sim 167.5$ eV1 was not observable in Figure S7a could indicate the lack of sulfur product oxidation at the ATP. The broad peak at $191.7 \mathrm{eV}$ is related to the appearance of the $\mathrm{B}-\mathrm{C}$ and $\mathrm{B}-\mathrm{O}$ bonds. ${ }^{3,4}$ In contrast, CPBA/4-ATP-free 3D AuAg nanohollows did not remarkably received the S 2P and B 1S signals.

a)

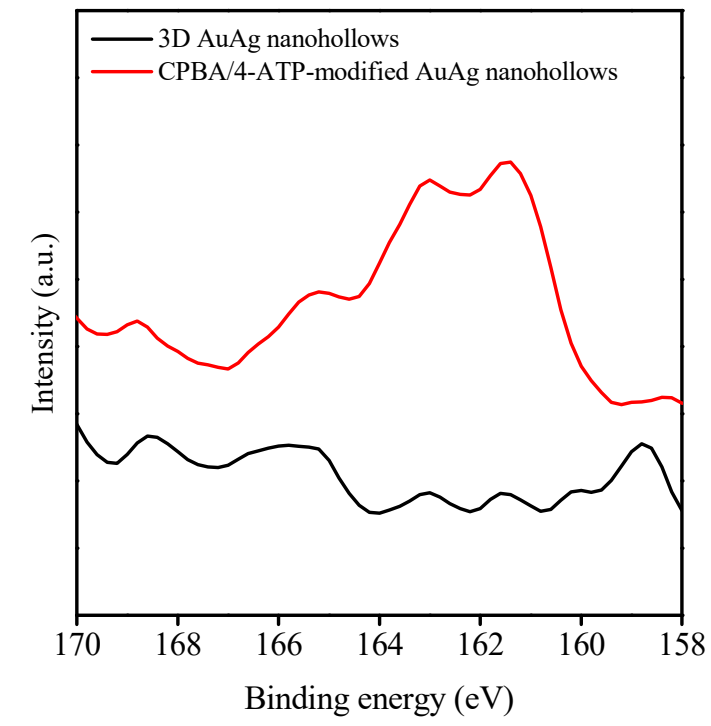

b)

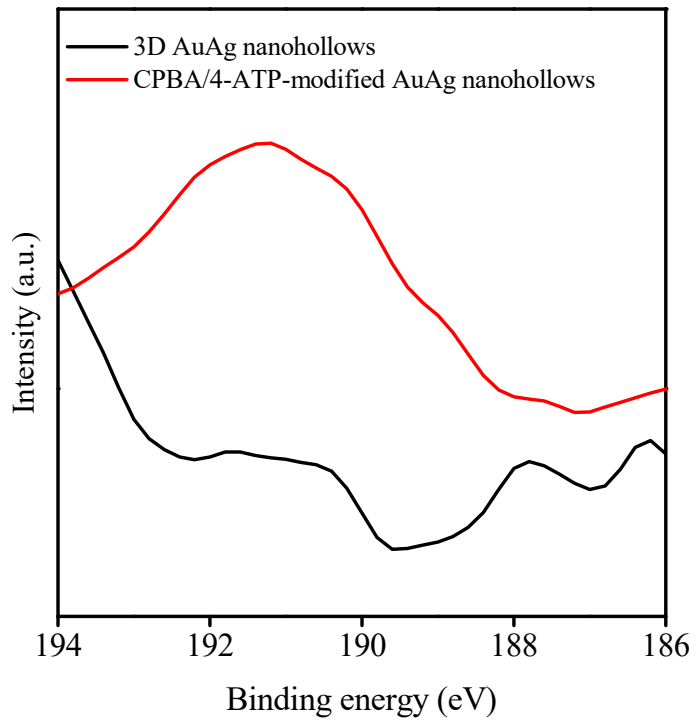

Figure S7. High-resolution XPS spectra for 3D AuAg nanohollows and CPBA/4-ATP modified $\mathrm{AuAg}$ nanohollows at a) sulfur $2 \mathrm{P}$ region and $\mathrm{b}$ ) boron $1 \mathrm{~S}$ region. 
a)
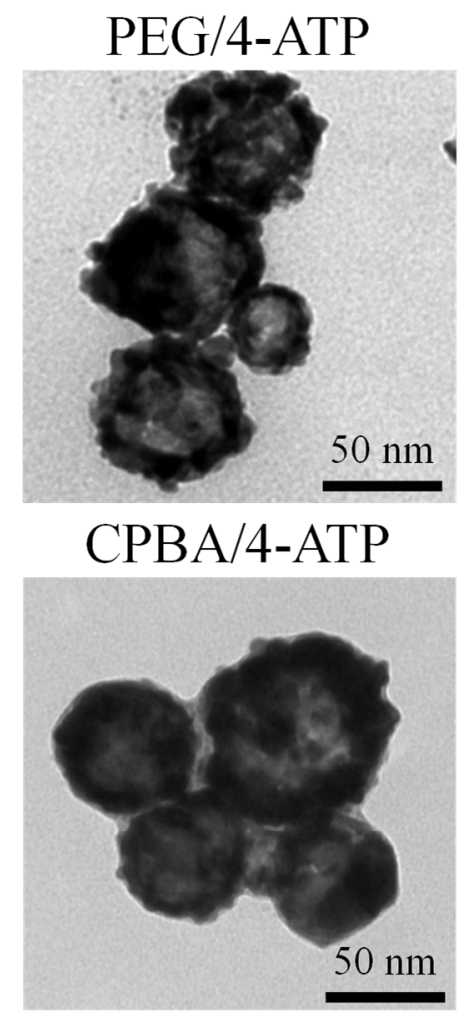

b)

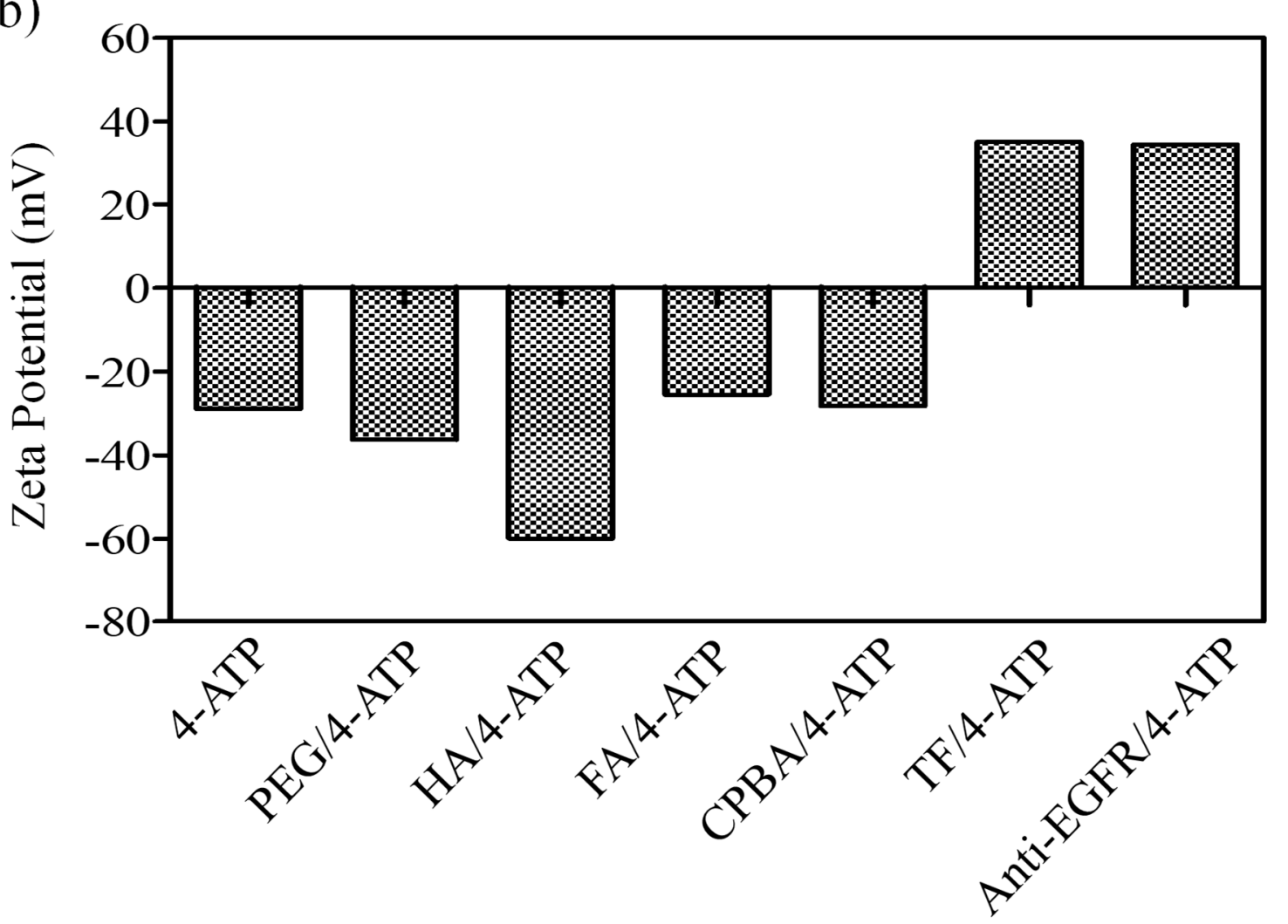

Figure S8. a) TEM images, and b) zeta potential of 4-ATP-modified AuAg nanohollows, PEG/4ATP-modified AuAg nanohollows, HA/4-ATP-modified AuAg nanohollows, FA/4-ATP-modified AuAg nanohollows, CPBA/4-ATP-modified AuAg nanohollows, TF/4-ATP-modified AuAg nanohollows, and anti-EGFR/4-ATP-modified AuAg nanohollows. 


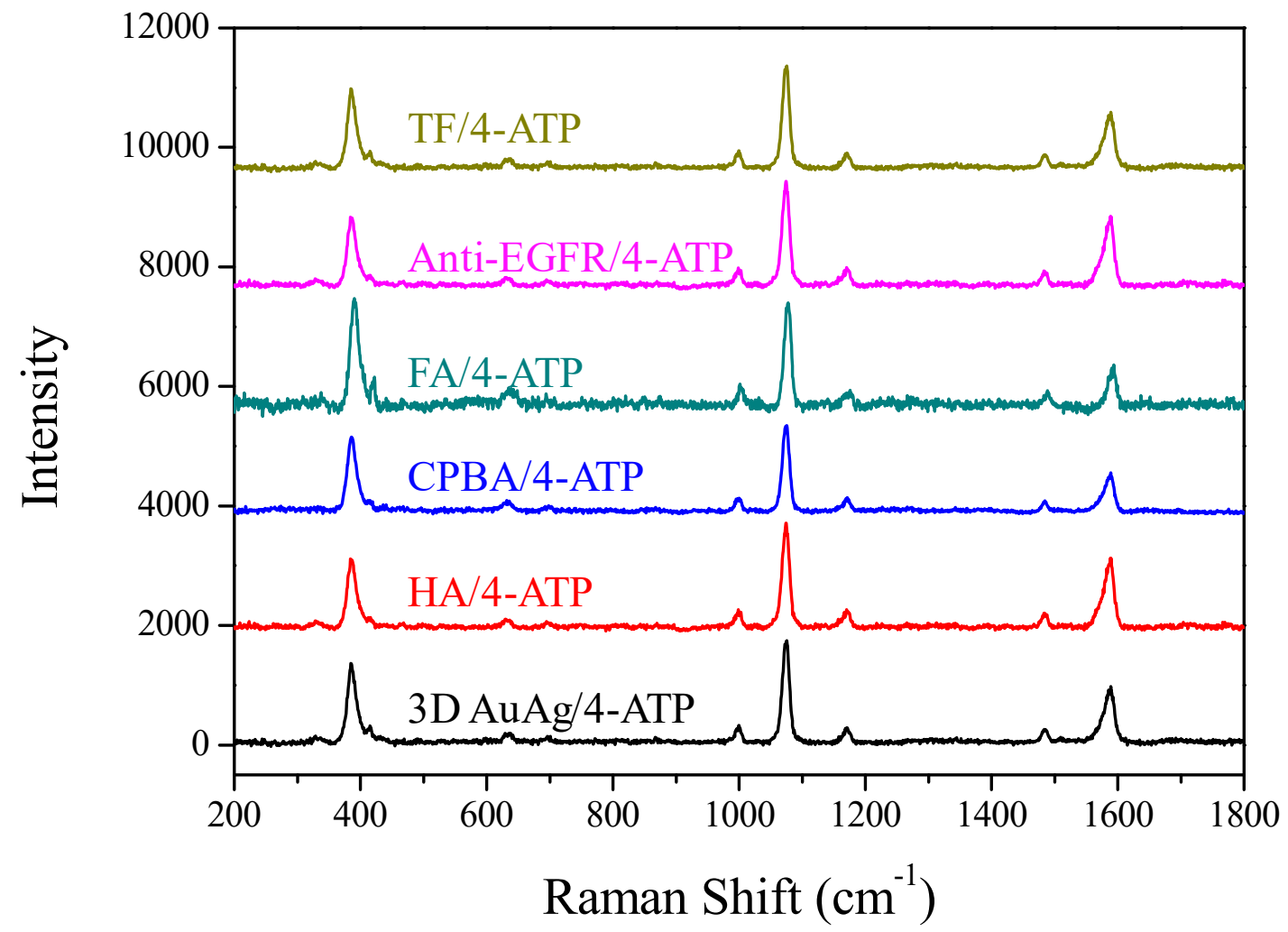

Figure S9. SERS measurements of the 3D AuAg nanohollows, HA/4-ATP-modified AuAg nanohollows, CPBA/4-ATP-modified AuAg nanohollows, FA/4-ATP-modified AuAg nanohollows, anti-EGFR/4-ATP-modified AuAg nanohollows, and TF/4-ATP-modified AuAg nanohollows. 

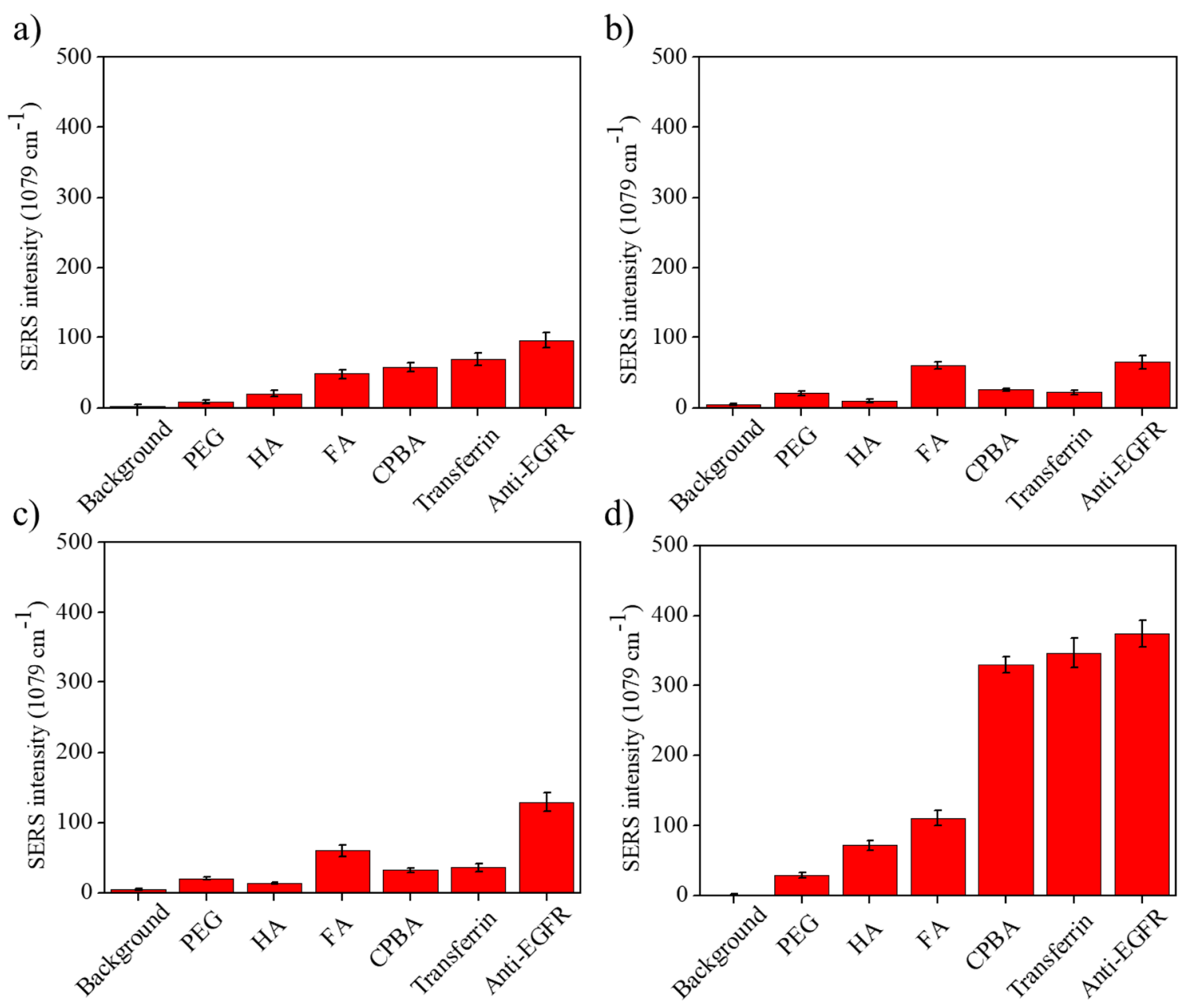

Figure S10. SERS measurements $(n=3)$ detected from the a) NIH/3T3 cells, b) SV-HUC1 cells, c) RT4 cells (low-grade), and d) T24 cells (high-grade) after reaction with ligand/4-ATP-modified AuAg nanohollows for 15 min. 10000 cells in each group were analyzed and data were obtained from three independent experiments. Data are presented as mean $\pm \mathrm{SD}$. 


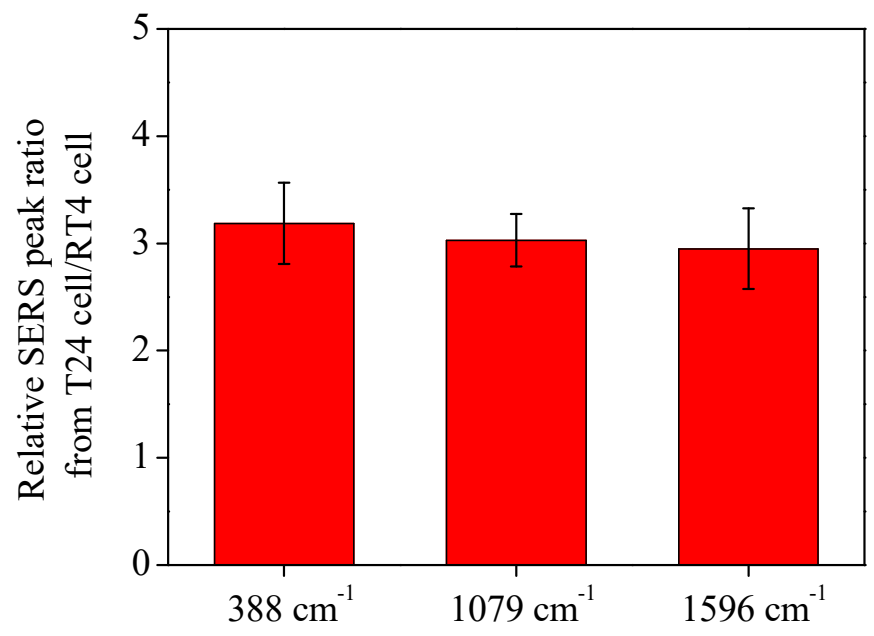

Figure S11. Comparison of the relative SERS peak intensity at $388 \mathrm{~cm}^{-1}, 1079 \mathrm{~cm}^{-1}$, and $1596 \mathrm{~cm}^{-}$ ${ }^{1}$ from the T24 cell/RT4 cell. Both cells were pre-treated with anti-EGFR/4-ATP-modified AuAg nanohollows for 15 min before SERS measurement. Data are presented as mean $\pm \mathrm{SD}, \mathrm{n}=3$. 
Additional CPBA concentration was performed to demonstrate that a $1.71 \mu \mathrm{g} / \mathrm{mL}$ CPBA is at optimal condition for SERS signal response and reproducible detection results. The SERS intensity slightly decreased and the corresponding standard derivation increased to these independent groups of the ligand/ATP-immobilized AuAg nano-hollows used at 1/4-foilds reaction concentration of CPBA molecule $(0.43 \mu \mathrm{g} / \mathrm{mL})$, as shown in Figure S12a. When $1 / 8$-folds amount of CPBA molecule $(0.21 \mu \mathrm{g} / \mathrm{mL})$ was applied in the same bio-conjugation reactions, the SERS peaks did not detect with respect to $<3$ of signal-to-noise ratio (Figure S12b).

In the present experiment, Figure S12c-e clearly showed the relative ligand binding curve of CPBA/4-ATP-modified AuAg nanohollows to CPBA/4-ATP-modified AuAg nanohollows and anti-EGFR/4-ATP-modified AuAg nanohollows. To improve the analysis precision, the ongoing work is necessary to explore the optimal ligand binding conditions, such as nanoparticle solution volume in shortening the ligand-to-cell binding time, reaction temperature of nanoparticle-cell interaction to prevent from the endocytosis pathway, the same targeted ligand number onto the SERS probes, and addition of the anti-fouling layer such as PEG and BSA to avoid the nonspecific adsorption.

a)

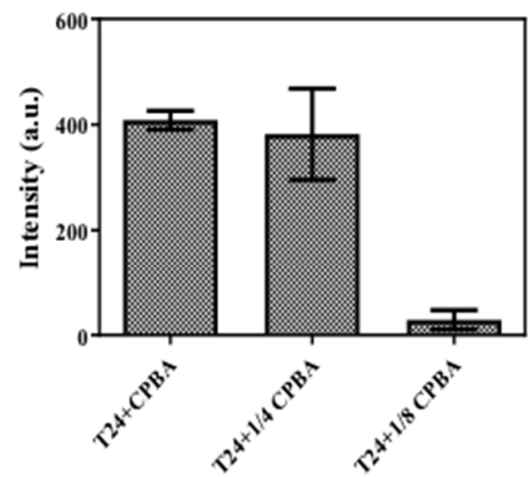

c)

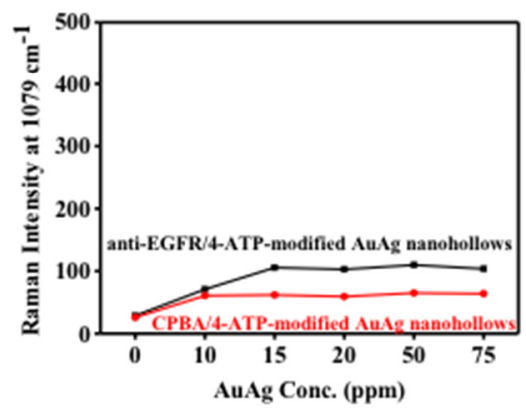

b)

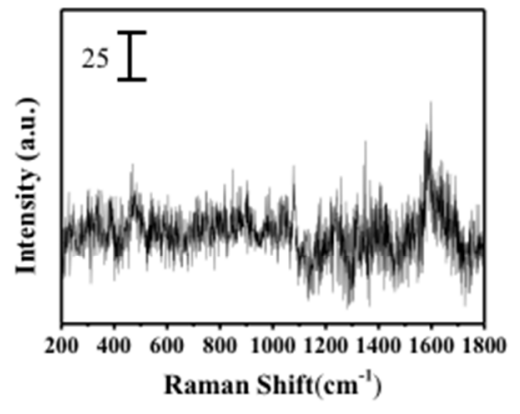

e)

Figure S12. a) Comparison of the SERS intensity from the T24 cells $(n=3)$ treated with CPBA/ATPimmobilized $\mathrm{AuAg}$ nanohollows, prepared at $1.71 \mathrm{ug} / \mathrm{mL}, 0.43 \mathrm{ug} / \mathrm{mL}$, and $0.21 \mathrm{ug} / \mathrm{mL}$ of CPAB molecules. b) SERS spectrum of the T24 cells treated with CPBA $(0.21 \mathrm{mg} / \mathrm{mL}) /$ ATP-immobilized $\mathrm{AuAg}$ nanohollows. The concentration-dependent SERS detections after 15 mins reaction with CPBA/4-ATP-modified AuAg nanohollows and anti-EGFR/4-ATP-modified AuAg nanohollows from c) NIH/3T3 cells, d) RT4 cells (low-grade), and e) T24 cells (high-grade). 
To understand the laser power threshold in photodamage of particle-targeted T24 cells, we examined the power-dependent photodestruction of these SERS-imaged cancer cells after a 4-h particle-cell interaction. A $785-\mathrm{nm}$ laser with $4-30 \mathrm{~mW}$ power through a $100 \mathrm{X}$ objective was employed using an irradiation time between 0 and $60 \mathrm{~min}$. We found that the cell morphology of the AuAg nanohollow-free group was unspoiled at 10X magnification of the laser power $(40 \mathrm{~mW})$ (Figure S13a). To investigate the introduction of a "wound" to the living cells, we exposed T24 cells to a 785-nm diode laser of $16 \mathrm{~mW}$. The phenomenon of membrane swelling ${ }^{5-7}$ and vacuoles was observed after $10 \mathrm{~min}$ irradiation (Figure S13c). Such light-stimulated reaction can be used to increase the size of the vacuole on the surface of each cell as a function of the laser irradiation time, thus injuring the plasma membrane to develop necrotic cells. ${ }^{5}$

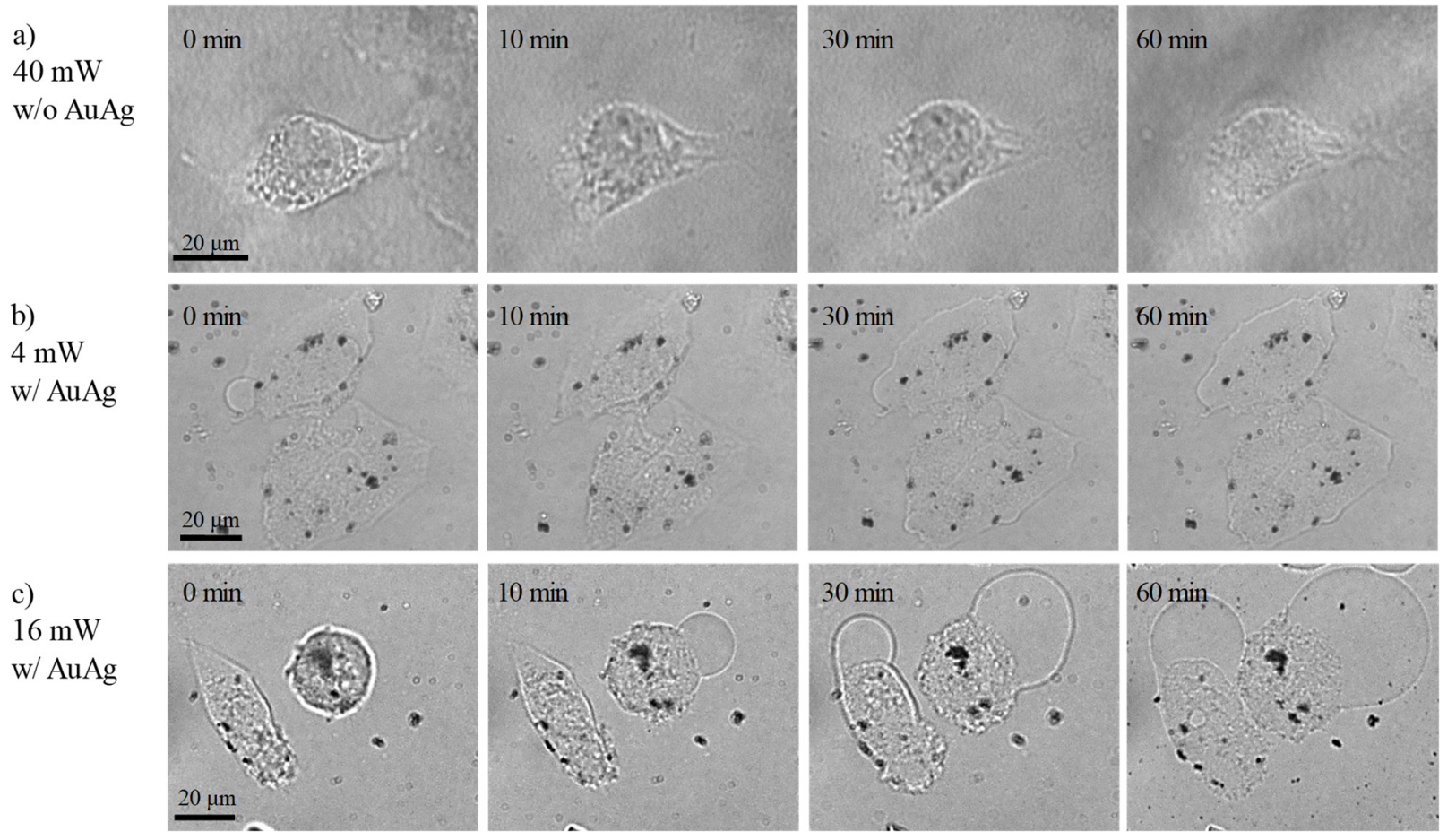

Figure 13. Photographs of the T24 cells a) that received $40 \mathrm{~mW}$ of $785-\mathrm{nm}$ laser power from $0-60$ min and T24 cells treated with 35 ppm[Ag] CPBA/4-ATP-modified AuAg nanohollows plus b) 4 $\mathrm{mW}$ of 785-nm laser power or c) $16 \mathrm{~mW}$ of 785-nm laser power from 0-60 min. 
Additionally, as the laser power was increased to $30 \mathrm{~mW}$, the complete photoablation of T24 cells under the focused spot was obtained at $60 \mathrm{~min}$ (Figure S14), in contrast to the formation of cell membrane damage by the $16 \mathrm{~mW}$ laser after the same amount of time. Higher photostability was revealed when the $3 \mathrm{~T} 3$ cells and SV-HUC1 cells treated with 35 ppm[Ag] CPBA/4-ATP-modified AuAg nanohollows and $785 \mathrm{~nm}$ laser at $40 \mathrm{~mW}$ exposure (data not shown).
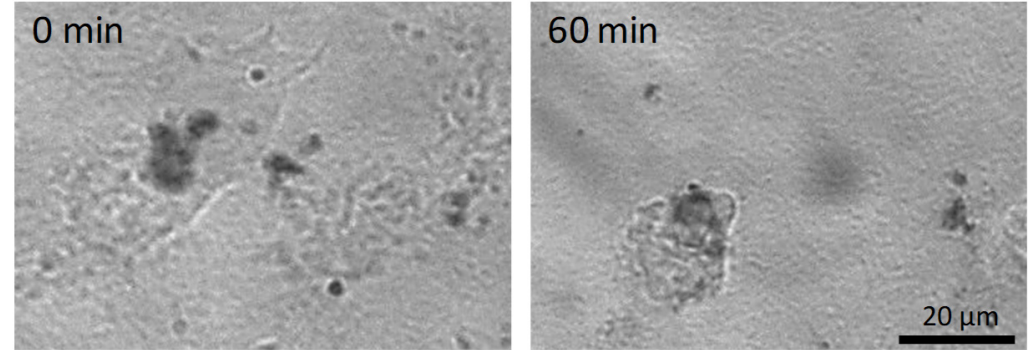

Figure S14. Images of T24 cells treated with 35 ppm $[\mathrm{Ag}]$ CPBA/4-ATP-modified AuAg nanohollows and T24 cells that received $30 \mathrm{~mW}$ of 785-nm laser power from 0-60 min. 
The MTT assay indicated that the T24 cells with the combined treatment of CPBA/4-ATP-modified AuAg nanohollows and 16-30 $\mathrm{mW}$ laser power for $10 \mathrm{~min}$ exhibited decreased viability by $\sim 15 \%$, which was in agreement with the image observation of the cells that suffered from dramatic photodamage. In contrast, increased viability was observed with short $(5 \mathrm{~min}$ ) laser-activated photothermal therapy, increasing by $74 \%$ with $16 \mathrm{~mW}$ and by $46 \%$ with $30 \mathrm{~mW}$. These results from our micro-Raman integrated targeted/labeled AuAg nanodevice readily gave new insight into the rational use of safe imaging-guided tracking to examine aggregative cancerous cells for effective downstream application of photothermal cancer therapy.

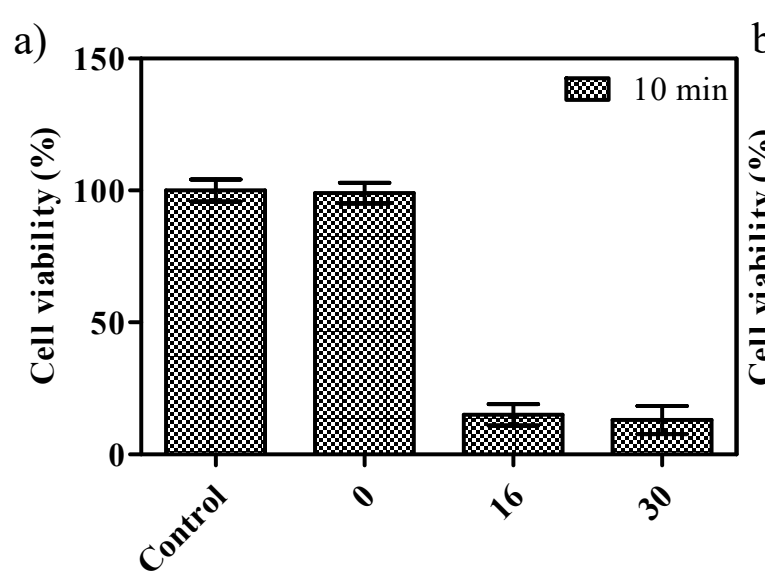

Laser power $(\mathrm{mW})$

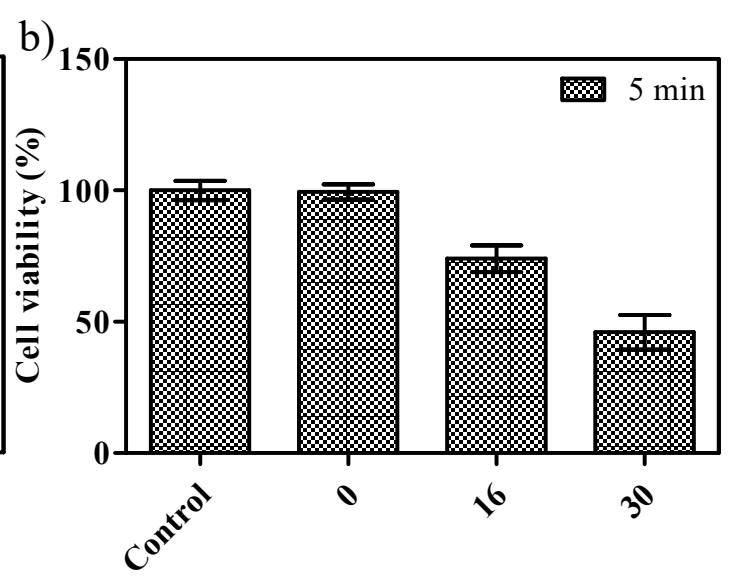

Laser power $(\mathrm{mW})$

Figure S15. MTT assay measurements of the T24 cells treated with CPBA/4-ATP-modified AuAg nanohollows and 16-30 $\mathrm{mW}$ of 785-nm laser power at a) $10 \mathrm{~min}$ and b) $5 \mathrm{~min}$. After irradiation, another $24 \mathrm{~h}$ of culture time was performed prior to MTT analysis. 

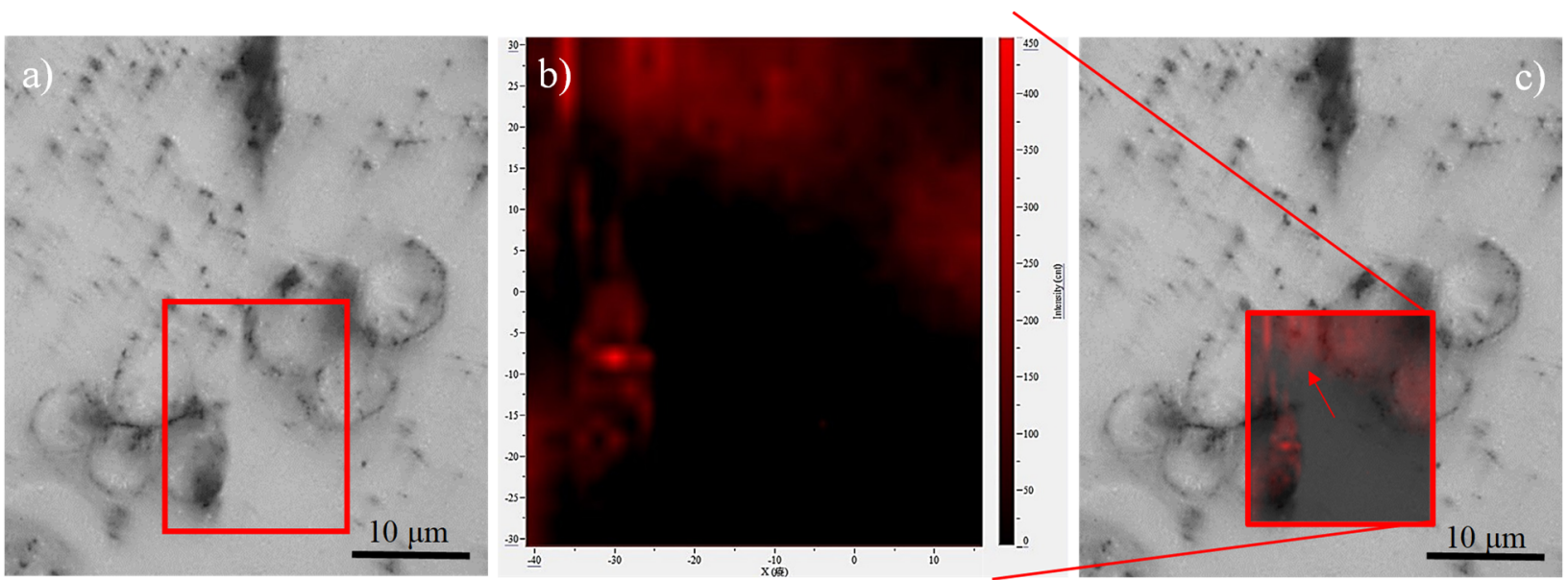

Figure S16. a) Bright field image, b) SERS mapping and c) SERS-bright field merged image of T24 cells incubated with CPBA/4-ATP-modified 3D AuAg nanohollows (20 ppm $[\mathrm{Ag}])$ at $37^{\circ} \mathrm{C}$ for $1 \mathrm{hr}$. The SERS mapping image was constructed from the Raman peak at $1079 \mathrm{~cm}^{-1}$ for the selected zone (red-square marked area) with point-to-point x-y scanning of the cancer cell particles in urine. The red arrow indicates the membrane fragments from destructed cells. 
Although the specific receptor proteins on bladder cells, HeLa cell, and A549 cells surfaces were general agreement with the enrichment in the relation with the malignant degrees of cancer cells, ${ }^{8}$ their corresponding targeted species specifically may suffer from the variability during the dynamic reaction regions between the antigens and targeted molecules in the biophysics processes (i.e., orientation and thermodynamic stability and flexibility characteristics of antibody). Therefore, the time-course responsive signals from these different bio-conjugated biomarkers are necessary to perform and will be carried out in our follow-up paper.

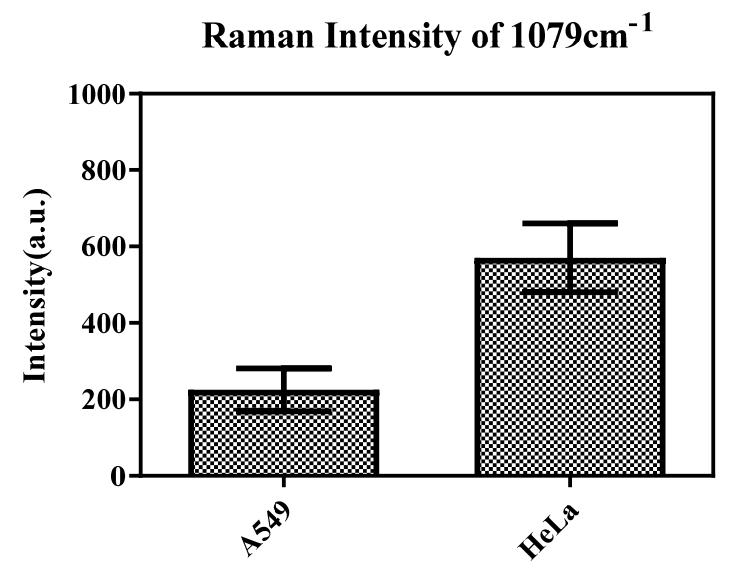

Figure S17. Comparison of the SERS intensity from the A549 cells and HeLa cells $(n=3)$ treated with FA/ATP-immobilized AuAg nanohollows. Data were obtained from three independent experiments. Data are presented as mean \pm SD. 

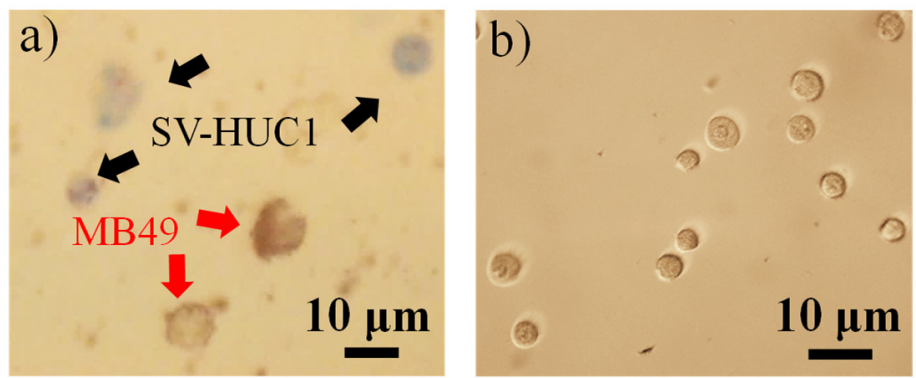

Figure S18. In vitro detection of normal cell and bladder cancer cell mixture. a) Image of the mixture of MB 49 cells (red arrow) and methylene blue-stained SV-HUC1 cells (blue cells, black arrow) after incubation with $20 \mathrm{ppm}[\mathrm{Ag}]$ 4-ATP/CPBA-modified AuAg nanohollows. b) Image of MB49 cells after incubation with 20 ppm ${ }_{[\mathrm{Ag}]}$ 4-ATP/PEG-modified AuAg nanohollow. 

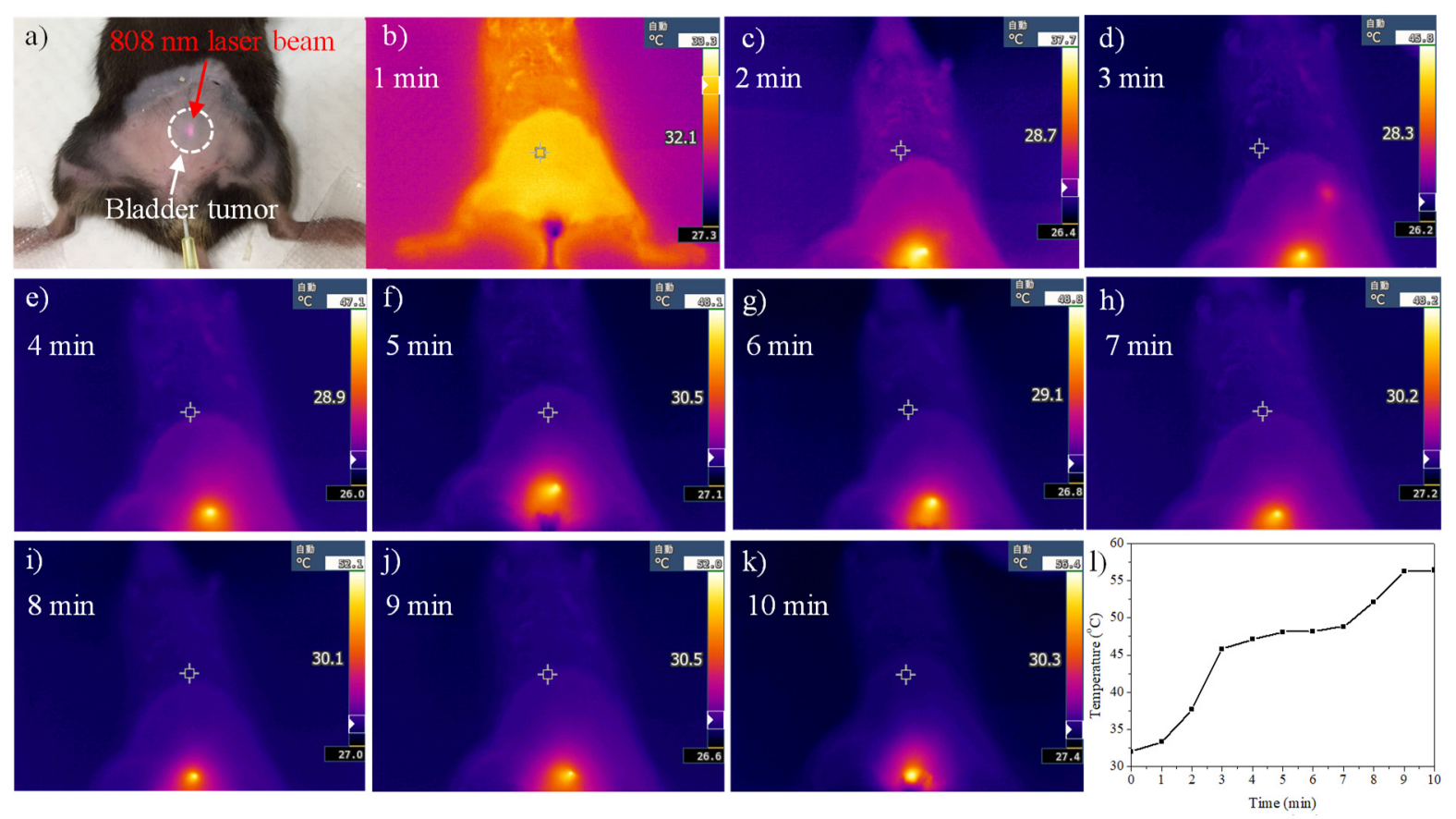

Figure S19. a) Photograph and b-k) thermogram images of MB49 tumor bearing mice before and after the $808 \mathrm{~nm}$ laser irradiation for $10 \mathrm{~min}$. 1) Temperature changes of tumor as a function of the irradiation time. $0.1 \mathrm{~mL}$ of CPBA/4-ATP-modified 3D AuAg nanohollow (100 ppm[Ag] $)$ was instilled into the mouse bladder cavity for the intravesical treatment $(1 \mathrm{~h})$. After $1 \mathrm{~h}-\mathrm{AuAg}$ nanohollow retention in the mouse bladder cavity, the AuAg nanohollow was drained from the bladder and the non-specific binding was removed by gently washing the bladder cavity three times with warm PBS. The mouse bladder was irradiated with $808 \mathrm{~nm}$ laser at a power density of 1.75 $\mathrm{W} / \mathrm{cm}^{2}(365 \mathrm{~mW})$ for $10 \mathrm{~min}$ at a fixed distance from the tip of laser fiber to the skin surface (1 $\mathrm{cm})$. 


\section{REFERENCE}

(1) Huang, C.-C.; Lin, P.-H.; Lee, C.-W. OFF/ON Galvanic Replacement Reaction for Preparing Divergent AuAg Nano-Hollows as a SERS-Visualized Drug Delivery System in Targeted Photodynamic Therapy. RSC Adv. 2016, 6, 64494-64498.

(2) Rosario-Castro, B. I.; Fachini, E. R.; Hernández, J.; Pérez-Davis, M. E.; Cabrera, C. R. Electrochemical and Surface Characterization of 4-Aminothiophenol Adsorption at Polycrystalline Platinum Electrodes. Langmuir 2006, 22, 6102-6108.

(3) Gao, B.; Zhao, F.; Miao, Y.; Min, H.; Xu, L.; Huang, C. Boron- and Nitrogen-Doped Photoluminescent Polymer Carbon Nanoparticles as Nanosensors for Imaging Detection of $\mathrm{Cu}^{2+}$ and Biothiols in Living Cells. RSC Adv. 2017, 7, 47654-47661.

(4) Liang, M.; Ren, Y.; Zhang, H.; Ma, Y.; Niu, X.; Chen, X. One-Step Synthesis of Nitrogen, Boron Do-Doped Fluorescent Carbon Nanoparticles for Glucose Detection. Luminescence 2017, 32, 1031-1038.

(5) Melamed, J. R.; Edelstein, R. S.; Day, E. S. Elucidating the Fundamental Mechanisms of Cell Death Triggered by Photothermal Therapy. ACS Nano 2015, 9, 6-11.

(6) Xu, S.; Olenyuk, B. Z.; Okamoto, C. T.; Hamm-Alvarez, S. F. Targeting Receptor-Mediated Endocytotic Pathways with Nanoparticles: Rationale and Advances. Adv. Drug Deliv. Rev. 2013, 65, 121-138.

(7) Abdelhalim, M. A.; Jarrar, B. M. Gold Nanoparticles Induced Cloudy Swelling to Hydropic Degeneration, Cytoplasmic Hyaline Vacuolation, Polymorphism, Binucleation, Karyopyknosis, Karyolysis, Karyorrhexis and Necrosis in the Liver. Lipids Health Dis. 2011, 10, 166.

(8) Ledermann, J. A.; Canevari, S.; Thigpen, T. Targeting the Folate Receptor: Diagnostic and Therapeutic Approaches to Personalize Cancer Treatments. Ann. Oncol. 2015, 26, 2034-2043. 